\title{
Inserción laboral de inmigrantes mexicanas calificadas en Estados Unidos - 2018
}

\section{Labor market insertion of qualified Mexican immigrant women in the United States - 2018}

María Inés Barrios de la $\mathrm{O}^{*}$

Recibido: Septiembre 2020 Aceptado: Diciembre 2020

Palabras Clave

Inserción laboral

Mujeres migrantes,

Migración calificada

Estados Unidos

\section{Keywords}

Labor market insertion

Women migrant

Skilled immigrants

United States
El fenómeno migratorio México-Estados Unidos es considerado como uno de los corredores migratorios más relevantes a nivel mundial, esto debido a su larga data, volumen y dinámica. A través del tiempo el perfil migratorio de mexicanos que se desplazan hacia el vecino país del norte a variado, tomando diferentes características y atributos. En las recientes décadas, se ha identificado que un perfil que se destaca en el stock de inmigrantes mexicanos en Estados Unidos ha sido el de las mujeres, el cual ha incrementado de manera significativa, particularmente de aquellas que cuentan con estudios de licenciatura y posgrado. Actualmente, radican en Estados Unidos alrededor de 380,000 mexicanas con altos niveles educativos, de las cuales, el 68.9 por ciento se encuentran insertas en el mercado de trabajo. En este sentido, el objetivo del presente artículo es mostrar un panorama de la inserción laboral de las migrantes mexicanas calificadas en el mercado laboral estadounidense.

\section{Abstract}

The Mexico-United States migratory phenomenon is considered one of the most relevant migratory corridors in the world, due to its long history, volume and dynamics. Through time, the migratory profile of Mexicans who move to the neighboring northern country has varied, taking on different characteristics and attributes. In recent decades, it has been identified that a profile that stands out in the stock of Mexican immigrants in the united states has been that of women, which has increased significantly, particularly of those with undergraduate and graduate studies. Currently, around 380,000 Mexican women with high educational levels live in the United States, of which 68.9 percent are inserted in the labor market. In this sense, the objective of this article is to show a panorama of the labor insertion of skilled Mexican immigrants in the US labor market.

\section{Introducción}

Entre las características identificadas del sistema económico globalizado han sido la división del trabajo, las estructuras y los procesos de reclutamiento de trabajo, así como el desarrollo de algunas economías basadas en el conocimiento y la innovación. Este contexto ha ocasionado que algunos países tengan una alta demanda de mano de obra calificada para sostener sus ritmos de crecimiento y desarrollo económico. De acuerdo con el Informe sobre las Migraciones en el Mundo de 2018, dos tercios del flujo migratorio internacional corresponden a migrantes económicos o trabajadores, de los cuales cerca del 45 por ciento son mujeres. Estos datos, analizados bajo el contexto de la globalización, nos

\footnotetext{
* Doctora en Estudios de Migración y coordinadora académica de la Especialidad en Migración Internacional y Maestría en Estudios de Migración Internacional deEl Colegio de la Frontera Norte.Contacto: innes.barrios@gmail.com
} 
brindan pinceladas de líneas de investigación para reflexionar sobre la importancia que tienen las mujeres migrantes dentro de los mercados laborales globales. De manera particular, se han identificado algunos estudios de migración que consideran las dimensiones de trabajo y género, los cuales se han centrado en los procesos de inserción laboral, así como el papel que poseen las mujeres dentro de las estructuras ocupacionales de los mercados de trabajo. Sin embargo, Kofman (2000) señala que al tratar de analizar los procesos de inserción laboral que tienen las inmigrantes calificadas, mujeres con grados educativos de licenciatura o posgrado, dentro de las estructuras de los mercados de trabajo, éstos han sido ignorados en muchas de las agendas de investigación, puesto que el papel laboral de las inmigrantes calificadas suele no evidenciarse al considerar a las mujeres inmigrantes como un todo y no hacer énfasis en las subpoblaciones.

El argumento de Kofman es relevante colocarlo a la luz de esta investigación, ya que las características o atributos suelen ser inherentes a las mujeres migrantes, los cuales llegan a fungir como determinantes de sus procesos de inserción laboral. Este es el caso de las inmigrantes que cuentan con un grado o posgrado académico, por poseer un nivel educativo superior a otras inmigrantes las lleva a considerarse como migrantes calificadas, lo que las diferencia del resto del stock de mujeres inmigrantes. La literatura desarrollada desde el enfoque teórico de las ciudades globales, el cual ha sido uno de los marcos de referencia para el análisis de los procesos migratorios de personas calificadas hacia países desarrollados, se ha centrado, particularmente, en el papel que tienen las inmigrantes dentro de los sectores económicos informales, como lo son los domésticos o de cuidados a pesar de que éstas sean calificadas. Este enfoque sobre la participación laboral en los mercado informales se ha producido debido a que internacionalmente hay una sobrerrepresentación y estigmatización de la participación de las mujeres en mencionados nichos laborales, lo que promueve a una invisibilización de las migrantes en ocupaciones calificadas, como lo son las ingenierías, matemáticas o ciencias bioquímicas, caso contrario a los hombres, los cuales, a través de la literatura se ha evidenciado que se emplean, particularmente, en puestos laborales e industrias basadas en el conocimiento o altamente calificadas (Kofman, 2003). Lo anterior, llega a reflejar que en los análisis y estudios de la migración calificada aún sigue prevaleciendo una noción androcéntrica y que los avances que se han construido durante las últimas décadas del siglo XX e inicios del siglo XXI, dentro de las diferentes áreas del conocimiento, como las ciencias sociales, particularmente en los estudios de migración, para visibilizar el rol de las mujeres, no aplica para la migración calificada, puesto que la ausencia de una perspectiva de género desdibuja la importancia que tienen las inmigrantes con alto nivel académico dentro de los mercados laborales de las comunidades de destino.
En el caso de la migración México-Estados Unidos, se ha vislumbrado que desde mediados del siglo $\mathrm{XX}$ se identifica la presencia y participación de inmigrantes mexicanos calificados en el mercado laboral estadounidense. Esto debido a que Estados Unidos es considerado como uno de los países lideres de las economías del conocimiento e innovación, lo cual ha llevado a valorar y demandar cada vez más capital humano para sostener las nuevas formas de producción (Aragonés y Salgado, 2015). Sin embargo, al analizar el papel de las inmigrantes mexicanas calificadas en el mercado laboral estadounidense se ha identificado que éstas no siempre se insertan en ocupaciones profesionales, Ramírez y Gandini (2016) mencionan que: “ [...] las migrantes mexicanas suelen insertarse laboralmente en ocupaciones que no siempre demandan los conocimientos, las habilidades y las capacidades desarrolladas durante su formación profesional, por lo que muchas de ellas sufren o enfrentan el problema de la descualificación o subutilización de capacidades, situación que es menos común entre las nativas y otras inmigrantes" (Ramírez y Gandini, 2016:52).

A partir del contexto planteado anteriormente y con la finalidad de poder explorar las hipótesis planteadas por Kofman (2003) y por Ramírez y Gandini (2016), el objetivo de este artículo es analizar los patrones de inserción laboral de las inmigrantes mexicanas calificadas en el mercado laboral estadounidense. Para ello se realiza un análisis estadístico descriptivo, con base en la información obtenida en la American Community Survey (ACS), 2018.

Este artículo se encuentra estructurado en tres secciones. En la primera sección se realiza un análisis sobre el papel que han tenido las mujeres en la migración particularmente las migrantes calificadas y su participación en los mercados de trabajo. En la segunda sección se presenta un análisis descriptivo sobre el comportamiento de la migración calificadas de mexicanos en el stock migratorio que se encuentra en Estados Unidos, así como la participación las migrantes en el mercado laboral. Finalmente, en la tercera sección se presentan algunas reflexiones y conclusiones de la presente investigación.

\section{Mujeres, migración calificada y mercado laboral}

El enfoque de género, así como el papel que poseen las mujeres en los flujos migratorios han permanecido ajenos a la mayoría de las teorías sobre migración internacional. Desde los enfoques teóricos clásicos hasta las teorías y perspectivas analíticas integracionistas no han logrado reconocer que la experiencia migratoria de las mujeres es diferente a la de los varones, ya que la gran mayoría se enfocan a explicar que la movilidad humana obedece a procesos macroestructurales de ordenes económico y político (Kanaiapuni, 2000). En este tenor, Pedraza (1991) argumenta que los enfoques teóricos que son utilizados para abordar los estudios de la 
migración han carecido, a través de tiempo, de un enfoque de género, por lo que la explicación del fenómeno migratorio ha sido falto e incapaz de proporcionar una comprensión real de las experiencias migratorias de hombres y mujeres. Incluso en la actualidad, muchos de los argumentos centrales de perspectivas analíticas que explican migración internacional, han estereotipado a la mujer, sosteniendo que la migración femenina se produce por ser sujetos dependientes, esposas y madres en un contexto de reunificación familiar (Pessar, 1986).

Durante las tres últimas décadas y con la finalidad de mostrar el rol de las mujeres en las migraciones internacionales, Hondaneu-Sotelo (2007) ha sostenido un énfasis de que el carácter laboral de la migración femenina es parte fundamental de la construcción de identidad de género desde un proceso migratorio. La inclusión del género como perspectiva analítica en los estudios de las migraciones contemporáneas es resultado de la necesidad de entender la presencia de las mujeres y el aumento de su participación en los procesos movilidad humana. Arias (2013), menciona que la migración femenina obedece a factores vinculados a la subordinación y a la falta de derechos en los sistemas de reproducción social patriarcales, además que la migración es un escape de las conductas masculinas que agreden su condición de mujer en las sociedades de origen.

Uno de los tópicos de mayor relevancia en los estudios e investigaciones que versan sobre la migración femenina se enfocan en el mercado laboral, así como en la participación económicamente activa las mujeres migrantes. La feminización de las migraciones, como se le denomina desde inicios del siglo XXI, se produce en un contexto ambiguo, en donde las características contemporáneas obedecen a las alteraciones de la economía global, la cual es normada por una interdependencia de los mercados y por el aumento de la vulnerabilidad de las mujeres migrantes trabajadoras (Sassen, 2008). La introducción de las mujeres en el mercado laboral, así como en los procesos migratorios, son respuestas a los mayores índices de educación femenina y a otros impactos demográficos; tales como la disminución de la natalidad, la postergación de la maternidad o la reducción del número de hijos (Boyd y Grieco, 2003). En este sentido, se reconoce que los factores económicos son los de mayor explicación entre los vínculos de la globalización y la migración femenina, la cual coadyuva a que muchas mujeres inviertan en mayor capital humano, tal es el caso de la escolaridad y así obtener mejores condiciones de vida.

El tema particular de la migración calificada tiene sobre la escena de los estudios migración desde la década de los cincuenta. Empero, este desarrollo de investigaciones no ha sido sistemático, aunque se puede identificar que su mayor auge se presenta durante la década de los noventa. Si bien, se destaca que se han producido un cumulo de investigaciones sobre el tema, aún no se ha logrado llegar a un consenso sobre cómo definir a los migrantes calificados y qué aspectos o características son los que se deben considerar. En el debate que se ha desarrollado en la literatura se han identificado aspectos para catalogar a una persona como calificada, entre los cuales se destacan los niveles de escolaridad, la experiencia laboral y las habilidades profesionales. Iredale (1999) argumenta que los migrantes calificados son quienes poseen al menos un grado universitario o una amplia experiencia en un campo o nicho de mercado de trabajo determinado, Rafael Alarcón (2007) define que los migrantes calificados tiene que ser aquellos que poseen, al menos, cuatro años de escolaridad universitaria, mientras que para Batalova (2008) son los inmigrantes que cuentan con licenciatura o un grado académico superior y que ingresaron a Estados Unidos antes de los 25 años y en este mismo tenor, Calva (2014) alude a los migrantes calificados como aquellas personas que nacieron en México y que obtuvieron, al menos, un título universitario y que posteriormente emigraron a Estados Unidos. Para fines de este artículo de investigación, se considera como migrantes calificadas aquellas mexicanas que cuentan con, al menos, un titulo universitario, y que se encuentren laborando en el mercado laboral estadounidense.

Pizarro (2008) menciona que tanto la migración femenina como la migración calificada constituyen dos de los temas de mayor relevancia en los estudios de las migraciones internacionales contemporáneas. En el actual panorama de la globalización y transformaciones de las estructuras económicas, la participación de las mujeres en el mercado laboral ha ido al alza, producto de la demanda existe que ha llevado a una división sexual del trabajo, desarrollando, en ciertos contextos, un sistema de desigualdad por género, en donde la mujer se encuentra en subordinación en comparación con los varones (Boyd y Grieco, 2003). En este sentido, se comienzan a identificar nichos de mercados determinados, que si bien pueden beneficiar a algunas inmigrantes también pueden ser considerados barreras para algunas otras que tengan habilidades, atributos o altos niveles educativos, ocasionando una movilidad profesional descendente o una subutilización de capacidades. A partir del análisis realizado en la siguiente sección del artículo, se muestra cuál y cómo ha sido la participación laboral de las inmigrantes mexicanas calificadas en el mercado laboral estadounidense, y si es que al igual que las investigaciones referida, los atributos correspondientes a sus niveles educativos han beneficiado o perjudicado su inserción ocupacional.

\section{Inmigrantes mexicanas calificadas en el mercado laboral estadounidense}

Una de las particularidades del flujo migratorio de mexicanos hacía Estados Unidos, durante la década de los ochenta y 
principios de los noventa, es que se caracterizaba por estar conformado por hombres y mujeres de baja calificación. Empero, durante el primer quinquenio de la década de los noventa se acentúa que la migración calificada ha sido considerada como uno de los fenómenos emergentes, esto debido al acelerado crecimiento que comenzó a presentar, además de los impactos económicos y sociales que se emprendieron a generar las sociedades de origen y destino (Calva y Alarcón, 2015). También se destaca que el flujo ha presentado relevantes cambios, entre los que se destacan su volumen, sus tendencias, sus modalidades, así como el perfil sociodemográfico, identificando el de la participación de mujeres con altos niveles de escolaridad, mujeres calificadas (Ramírez y Gandini, 2016). En el cuadro 1 se puede observar cómo es que ha cambiado, a través del tiempo, la presencia de migrantes mexicanas calificadas en Estados Unidos. Se destaca que, aunque el porcentaje, en comparación a las mexicanas no calificadas, es muy bajo, su crecimiento ha sido mayor.

Desde el año 2000 al 2015 las tasas de crecimiento anual han sido duplicadas. Mientras que la presencia de mexicanas no calificadas en Estados Unidos tuvo una tasa de crecimiento anual de 4.6 por ciento, las mexicanas calificadas tuvieron una tasa de 8.1 por ciento, este patrón se mantiene para los siguientes dos quinquenios.

CUADRO 1

Comportamiento del stock de inmigrantes mexicanas calificadas en Estados Unidos, 2000-2018

\begin{tabular}{|c|c|c|c|c|c|c|c|c|}
\hline & \multicolumn{5}{|c|}{ Años } & \multicolumn{3}{|c|}{$\begin{array}{c}\text { Tasa de crecimiento } \\
\text { anual }\end{array}$} \\
\hline & 2000 & 2005 & 2010 & 2015 & 2018 & $\begin{array}{l}2000- \\
2005\end{array}$ & $\begin{array}{l}2005- \\
2010\end{array}$ & $\begin{array}{l}2010- \\
2015\end{array}$ \\
\hline Mexicanas & $3,224,764$ & $4,065,363$ & $4,817,902$ & $5,158,521$ & $5,396,569$ & 4.7 & 3.5 & 1.38 \\
\hline Calificadas & $4.34 \%$ & $5.08 \%$ & $5.92 \%$ & $6.93 \%$ & $6.97 \%$ & 8.1 & 6.7 & 4.61 \\
\hline $\begin{array}{l}\text { No } \\
\text { Calificadas }\end{array}$ & $95.66 \%$ & $94.92 \%$ & $94.08 \%$ & $93.07 \%$ & $93.05 \%$ & 4.6 & 3.3 & 1.16 \\
\hline
\end{tabular}

Fuente: Elaboración propia con base en la American Community Survey (IPUMS2018).

Como ya se ha señalado anteriormente, la inserción laboral es considerada como una de las dimensiones más significativas en los estudios de migración, debido a que ésta se vuelve una de las dimensiones que dan cuenta sobre los procesos socioeconómicos de los inmigrantes en las sociedades de destino. A través de los procesos de inserción laboral se logran identificar las ocupaciones en las que se encuentran los migrantes, los sectores e industrias económicas en donde se establecen, además que permite identificar, explorar y analizar las trayectorias laborales y la vinculación del desarrollo profesional en el mercado de trabajo.

Con base en la American Community Survey se puede identificar el estatus de la fuerza laboral de las migrantes mexicanas calificadas. En el cuadro 2 se muestra que la tasa de participación económica de estas inmigrantes es de $71.45 \%$, lo que indica que de cada cien inmigrantes mexicanas calificadas que se encuentran en la población económicamente activa, setenta realizan una actividad económica en el mercado laboral estadounidense. Esta tasa es similar a la que presentan las otras mujeres calificadas residentes en Estados Unidos. Aunque se destaca que las que tienen una mayor tasa de partición son las nativas, el hecho de no ser migrantes representa menores barreras para poder estar de manera activa en el mercado de trabajo. Otro de los aspectos relacionados con el estatus laboral de migrantes es la tasa de desempleo, la cual es del 3.57 por ciento, una de las tasas más bajas en comparación con otras poblaciones migrantes, como lo son las no calificadas. Uno de los resultados de mayor interés sobre las inmigrantes calificadas, es el alto porcentaje que se encuentra en inactividad económica, que es cerca del 28.55 por ciento, esto indica que de cada cien inmigrantes mexicanas que se encuentran en edad laboral 29 se consideran dentro de la población no económicamente activa ${ }^{1}$. Este último resultado da pie para reflexionar sobre la existencia de motivos por los cuales sucede esto, los cuales podrían obedecer a aspectos estructurales del mercado laboral o a determinantes relacionados con los roles de género.

CUADRO 2

\begin{tabular}{|c|c|c|}
\hline Situación laboral de inmigran & Intes mexicanas calificad & en Esta \\
\hline & Población en edad laboral & 376,141 \\
\hline $\mathrm{DF}$ & Empleadas & 249,606 \\
\hline $\mathbf{P E} t$ & Desempleadas & 9,242 \\
\hline & PNEA & 103,446 \\
\hline & Tasa de ocupación & $68.90 \%$ \\
\hline & sa de actividad económica & $71.45 \%$ \\
\hline & Tasa de desempleo & $3.57 \%$ \\
\hline Tasa d & de inactividad económica & $28.55 \%$ \\
\hline
\end{tabular}

Fuente: Elaboración propia con base en la American Community Survey (IPUMS2018).

Otro de los aspectos relevantes sobre la inserción laboral se relaciona con las ocupaciones en las que se encuentran insertas las inmigrantes. Esta dimensión representa una parte fundamental de la situación laboral que experimentan las mexicanas, ya que nos indica si las inmigrantes calificadas, más allá de estar o no insertas en el mercado de trabajo, se encuentran en una ocupación calificada o no, y aquí es precisamente en donde podemos vislumbrar si existe una subutilización de sus capacidades, habilidades y conocimientos

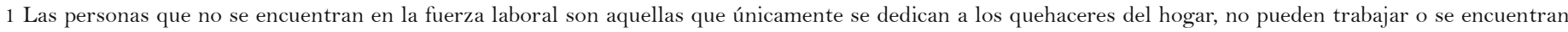
estudiando. 
proporcionados por su formación académica. En el cuadro 3 se muestra que el $11.6 \%$ de las inmigrantes mexicanas con licenciatura o posgrado son maestras de escuelas primarias o secundarias, mientras que el $4.6 \%$ son contadoras y auditoras y el $3.6 \%$ enfermeras registradas. Estas ocupaciones corresponden, en gran medida, a la formación con la que cuentan las inmigrantes, ya que gran parte de éstas obtuvieron su formación en áreas administrativas y de educación. Aunque también se puede identificar que el 3\% de las mexicanas calificadas se encuentran trabajando como sirvientas y trabajadoras de la limpieza, ocupación que no es considerada como calificada, ya que no requiere una formación académica de licenciatura o posgrado según la clasificación realizada por el Bureau of Labor Statistics de Estados Unidos.

\section{CUADRO 3}

Distribución de inmigrantes mexicanas calificadas por ocupaciones en el mercado laboral estadounidense, 2018

MUJERES

\begin{tabular}{lc}
\hline TOTAL DE INMIGRANTES & $\mathbf{1 0 0 \%}$ \\
\hline Maestros de escuela primaria y secundaria & $11.60 \%$ \\
Contadores y auditores & $4.60 \%$ \\
Enfermeras registradas & $3.60 \%$ \\
Secretarias y asistentes administrativas & $3.50 \%$ \\
Sirvientas y limpiadores de limpieza & $3.00 \%$ \\
Gerentes diversos & $2.90 \%$ \\
Trabajadoras sociales & $2.70 \%$ \\
Trabajadoras de cuidado de nuños & $2.60 \%$ \\
Maestras de secundaria & $2.40 \%$ \\
Maestros de preparatoria & $2.40 \%$ \\
Otras ocupaciones & $60.70 \%$ \\
\hline
\end{tabular}

Fuente: Elaboración propia con base en la American Community Survey (IPUMS2018).

Uno de los casos por resaltar o más emblemáticos sobre la inserción laboral de inmigrantes calificadas, es la que se desenvuelve entorno al sector salud en Estados Unidos, ya que su alta demanda de mano de obra obedece, particularmente, por el acelerado proceso de transición demográfica (Batalova, 2007). En los estudios de migración internacional, el contexto de la migración de enfermeras o personal enfocado al sector salud, ha sido considerado como uno de los referentes para el estudio de la institucionalización de las migraciones calificadas, ya que se ha desarrollado a partir de la existente relación entre diversas instituciones, así como la toma de decisiones de los individuos para migrar y los flujos migratorios internacionales (Massey, 2000). Para las inmigrantes mexicanas, a pesar de que la proporción de concentración en la ocupación de enfermeras registradas es baja en comparación a las que se encuentran en las áreas de educación y administración, se podría deducir, a partir de los resultados obtenidos por otras investigaciones (Rodríguez, 2004, Rosales, 2014; Hualde y Rosales, 2017), la existencia de un porcentaje mayor de enfermeras mexicanas que se sitúan en ocupaciones que posiblemente no correspondan a su formación académica o que se sitúen en trabajos no calificados, ya que para este grupo de inmigrantes se han reconocido barreras muy concretas para su inserción laboral, como lo son la ausencia de documentación migratoria requerida para ejercer su carrera profesional o el no reconocimiento de sus títulos o credenciales al ser ocupaciones con mayores regulaciones por parte de los estados (Rosales, 2014).

En el caso de las ocupaciones relacionadas con los servicios de limpieza o sirvientas, se identifica que son actividades laborales que no requieren alguna formación académica determinada. Investigaciones como las de Mollard y Umar (2012), Koffman (2013) y Castles, (2013) evidencían que la ausencia de algún reconocimiento o validación de experiencia profesiones y de habilidades adquiridas durante una formación académica obliga a muchas inmigrantes calificadas a laborar en actividades feminizadas y precarizadas, como es el caso del trabajo doméstico o de cuidados. Aunque más allá de caracterizarse por una precarización laboral, en contextos laborales de primer mundo estas ocupaciones son percibidas y consideradas como degradantes, dándoles un menor valor social, puesto que en varios contextos de países desarrollados las poblaciones nativas no están dispuestas a desempeñar dichas actividades (Hochschild, 2003).

Hasta este punto, se ha logrado identificar cuál es la situación laboral de las inmigrantes mexicanas calificadas y cuáles son las ocupaciones en las que se encuentran insertas. Sin embargo, un aspecto relevante a identificar en esta investigación es que, aunque las mexicanas se encuentren en ocupaciones que podrían considerarse como no calificadas, esto no las excluye de estar en una situación de deskilling. Rubin, et. al., (2009) señalan que el deskilling se presenta cuando las profesionistas se desempeñan en actividades o en sectores distintos a su formación educativa o en trabajos que requieren niveles profesionales o educativos que se encuentran por debajo de sus calificaciones adquiridas. El deskilling puede obedecer a distintos determinantes, los cuales se relacionan, particularmente, con el reconocimiento de credenciales, el dominio del idioma de la sociedad de destino o el estatus de residencia (Jungwirth, 2011).

En el cuadro 4 se muestra la distribución porcentual de las inmigrantes mexicanas calificadas por su condición en el mercado laboral estadounidense, en donde se identifica que el $44.92 \%$ de las inmigrantes mexicanas se encuentran en una situación de deskilling, mientras el $55 \%$ de éstas se encuentran en ocupaciones que requieren, al menos, el grado de licenciatura. 


\section{DOXA}

CUADRO 4

Inmigrantes mexicanas calificadas en deskilling, 2018

\begin{tabular}{lc}
\hline & Mexicanos \\
\hline & Mujeres \\
\cline { 2 - 2 } Total de personas & $\mathbf{1 0 0 \%}$ \\
En ocupaciones calificadas & $55.08 \%$ \\
En ocupaciones no calificadas & $44.92 \%$ \\
\hline
\end{tabular}

Fuente: Elaboración propia con base en la American Community Survey (IPUMS2018).

Uno de los aspectos que se debe de considerar al analizar estos datos, es que si bien, existe un porcentaje considerable de mexicanas calificadas en una ocupación calificada, es decir, en correspondencia con el grado o nivel formativo, esto no indica que exista correspondencia entre la ocupación y formación de las inmigrantes calificadas, ya que pueden existir divergencias entre estos dos elementos.

\section{Conclusiones}

El objetivo central de este artículo fue analizar la inserción laboral de las inmigrantes mexicanas calificadas que radican en Estados Unidos. A partir del análisis realizado se puede identificar que las mexicanas calificadas son uno de los grupos migratorios con mayor crecimiento en las recientes décadas, ya que sus tasas de crecimiento anual fueron muy superiores a las de otros migrantes, como es el caso de las mexicanas no calificadas, esto a pesar de que su representación proporcional es muy baja.

Uno de los principales aspectos que se pudieron evidenciar a través de esta investigación, fue la situación laboral que experimentan las inmigrantes calificadas, en donde se logró observar que presentan una tasa de inactividad económica cercana al treinta por ciento, lo que indica que de cada diez inmigrantes mexicanas que se encuentran en Estados Unidos, al menos tres no están trabajando ni buscando un trabajo. Ante este contexto, surge la pregunta del porqué se presenta esta situación, o si esto obedece al patrón que se ha mencionado en otras investigaciones, las cuales señalan que la participación de mujeres dentro del stock de inmigrantes en Estados Unidos es por una situación de reunificación familiar, actores dependientes de otros inmigrantes varones o porque simplemente se sitúan bajo un proyecto migratorio que no es el de ellas.

Una de las contribuciones de este artículo fue la identificación de la población inmigrante calificada que se encuentra en correspondencia entre su área de formación académica y su ocupación, ya que si bien se identificó un porcentaje considerable de inmigrantes mexicanas que se encontraban en un trabajo que requería un nivel de formación de licenciatura o posgrado, esto no garantizaba que existiera también una correspondencia con su campo de estudio,
pISSN: $2395-8758$

eISSN: 2594-2786 ya que en investigaciones se ha señalado la existencia de ingenieros, médicos o arquitectos que se desempeñan como educadores de educación básica. Si bien en este escenario se puede inferir en una correspondencia con nivel de formación en la actividad laboral desempeñada, ésta no concierne a su campo de estudio.

A partir del análisis aquí realizado, surgen interrogantes que pueden dar origen a nuevas exploraciones investigativas sobre el tema de la inserción laboral de inmigrantes calificadas, por ejemplo ¿por qué una proporción tan significativa de inmigrantes mexicanas calificadas no se encuentran insertas en el mercado laboral? ¿existen factores personales, familiares o contextuales que explican su inserción laboral en ocupaciones que no corresponden con su nivel de formación profesional? $\mathrm{O}$ ¿qué factores inciden en la situación de deskilling que presentan más del cuarenta por ciento de las inmigrantes mexicanas?

\section{Referencias}

Alarcón, R. (2007) "The free circulation of skilled migrants in North America” en Antoine Pécoud y Paul de Guchteneire (Eds.) Migration Without Bordes. Essays on the Free Movement of people. Paris: UNESCO Publishing y Oxford - New York Berhahn Books.

Aragonés, A. M. y U. Salgado (2015) La migración laboral MéxicoEstados Unidos veinte años del Tratado de Libre Comercio de América del Norte, en Revista Mexicana de Ciencias Políticas y Sociales, Año LX, núm. 224, pp. 279-314.

Batalova, J. (2007) "Foreign-Born Health-Care Workers in the United States", The online journal of Migration Policy Institute.

Batalova, J. y M. Fix (2008) Uneven Progress. The employment pathways of skilled immigrants in the United States, National Center on Immigrant Integration Policy.

Boyd, M. y E. Grieco (2003) Women and Migration: Incorporating Gander into International Migration Theory.

Calva, L. (2014) La migración calificada de mexicanos a Estados Unidos y su inserción en el mercado laboral. Tesis de doctorado en Ciencias Sociales con especialidad en Estudios Regionales. El Colegio de la Frontera Norte, A.C. México, $189 \mathrm{pp}$.

Calva, L. y R. Alarcón (2015) La integración laboral precaria de los inmigrantes mexicanos calificados en Estados Unidos al inicio del siglo XXI, en Papeles de Población, Vol. 21, número 83, pp. 9-39.

Castles, S. (2013) Migración, trabajo y derechos precarios: perspectiva histórica y actual, en Migración y desarrollo, vol.11, núm.20, pp. 8-42.

Hochschild, A. (2003) "Love and gold", en Ehrenreich, B. y 
Hochschild, A. (eds.) Global Woman, nannies, maids, and sex workers in the new economy, Nueva York: Henry Holt and Company, pp.15-30.

Hondagneu-Sotelo, P. (2007) "La incorporación del género a la migración: No sólo para feministas ni sólo para la familia”, En M. Ariza y A. Portes (Ed.). El país transnacional. Migración mexicana y cambio social a través de la frontera, pp.423-453, México D.F: Universidad Autónoma de México.

Hualde, A. y Y. Rosales (2017) profesionales que emigran: na comparación entre enfermeras e ingenieros mexicanos en Estados Unidos, en Sociedad, pp.-

Iredale, R. (1999) The need to import skilled personnel: Factors favoring and hide. International migration, Vol. 39, num. $7-26$

Kanaiapuni, S. (2000) "Reframing the Migration Question: An Analysis of Men, Women, and Gender in Mexico", Social Forces, North Carolina, The University of North Carolina Press, Vol. 78, No. 4, Jun 2000 Pp. 1311-1347.

Kofman, E. (2000) The invisibility of skilled female migrants and gender relations in studies of skilled migrant on in Europe, en International Journal of Population Geography, núm 6(1), pp. 45-59.

Kofman, E. (2003) Towards a gendered evaluation of (highly) skilled immigration in policies in Europe, en International Migration (IOM), vol. 52, Issue, 3, pp. 116, 128.

Mollard, B. y S. Umar (2012) "Gender, migration and deskilling - A broad review of the literature" en Crushed Hopes: Underemployment and deskilling among skilled migrant women, OIM, Switzerland, pp. 9-36.

Pedraza, S. (1991) "Women and migration: the social consequences of gender". Annual Review of Sociology, 17, 303-325.

Pessar, P. (1986) "The role gender in Dominican settlement in the United States", edits. Nash, June y Helen Safa, Women and Change in Latin America, Massachusetts, Massachusetts, Bergin \& Garvey, pp. 273- 294.

Ramírez, T. y L. Gandini (2016) “Trabajadoras calificadas: las mujeres mexicanas calificadas en el Mercado de trabajo estadounidense en perspectiva comparada”, en Revista latinoamericana de población, Año 10, núm. 19, juliodiciembre 2016, pp. 33-56.

Rodríguez, A. (2004) Programa de certificación para los enfermeros mexicanos en Estados Unidos. Caso de estudio: programa de preparación de profesionistas en enfermería mexicanos (tesis de maestría inédita). Universidad de las Américas: Puebla.

Rosales, Y. (2014) "La inmigración de enfermeras mexicanas en California, Procesos de inserción laboral dentro y fuera del sector salud". Tesis de Doctorado en Ciencias Sociales con Especialidad en Estudios Regionales. El Colegio de la Frontera Norte, A.C., México, 252pp.

Rubin, J., M. Rendall, L. Rabinovich, F. Tsang, C. Oranje-Nassau, B. Janta (2008) Migrant women in the European labour force Current situation and future prospects, Technical report prepared for the European Commission, RAND, 207pp.

Sassen, S. (2008) "Two stops in today's new Global geographies: shaping novel labor supplies and employment regimes". Santiago de Chile: CEPAL 